\title{
IMAGING THE DISK AROUND THE LUMINOUS YOUNG STAR LkH $\alpha 101$ WITH INFRARED INTERFEROMETRY
}

\author{
P. G. Tuthill, ${ }^{1}$ J. D. Monnier,${ }^{2}$ W. C. Danchi, ${ }^{3}$ D. D. S. Hale, ${ }^{4}$ and C. H. Townes ${ }^{4}$ \\ Received 2002 March 13; accepted 2002 June 8
}

\begin{abstract}
The Herbig Ae/Be star $\mathrm{LkH} \alpha 101$ has been imaged at high angular resolution at a number of wavelengths in the near-infrared (from 1 to $\sim 3 \mu \mathrm{m}$ ), using the Keck I Telescope, and also observed in the mid-infrared $(11.15 \mu \mathrm{m})$, using the UC Berkeley Infrared Spatial Interferometer (ISI). The resolved circular disk with a central hole or cavity reported by Tuthill, Monnier, \& Danchi is confirmed. This is consistent with an almost face-on view (inclination of $\lesssim 35^{\circ}$ ) onto a luminous pre-main-sequence or early main-sequence object surrounded by a massive circumstellar disk. With a multiple-epoch study spanning almost 4 years, relative motion of the binary companion has been detected, together with evidence of changes in the brightness distribution of the central disk/star. The resolution of the $\mathrm{LkH} \alpha 101$ disk by ISI mid-infrared interferometry constitutes the first such measurement of a young stellar object in this wavelength region. The angular size was found to increase only slowly from 1.6 to $11.15 \mu \mathrm{m}$, inconsistent with standard power-law temperature profiles usually encountered in the literature, supporting instead models with a hot inner cavity and relatively rapid transition to a cool or tenuous outer disk. The radius of the dust-free inner cavity is consistent with a model of sublimation of dust in equilibrium with the stellar radiation field. Measurements from interferometry have been combined with published photometry, enabling an investigation of the energetics and fundamental properties of this prototypical system.
\end{abstract}

Subject headings: circumstellar matter — infrared: stars — stars: individual ( $\mathrm{LkH} \alpha 101)$ — stars: mass loss - techniques: interferometric

\section{INTRODUCTION}

Among the brightest young stellar objects in the infrared sky, $\mathrm{LkH} \alpha 101$ is thought to be a transitional object which is on (or nearly on) the main sequence, but is still surrounded by a massive circumstellar disk. Following active accretion, hot stars are expected to pass through a brief phase in which the remnant accretion disk, which retains up to 0.3 times the mass of the central star (Shu et al. 1990; Hollenbach et al. 1994), is sculpted and eventually dissipated by the radiation and wind from the newborn star. The structure of these disks has been speculative, with uncertainty surrounding characteristic sizes and the existence of an inner cavity.

Images of young circumstellar disks in Orion (O'Dell, Wen, \& Hu 1993; McCaughrean \& O'Dell 1996) and remnant, or fossil, disks at large distances from the star around "Vega-type" sources, such as $\beta$ Pic (Smith \& Terrile 1984), have been seen in reflected light or silhouetted against bright nebulosity. This work has attracted intense interest for, as first hypothesized by Kant (1755), it is likely that our own solar system grew out of such a flattened primordial nebula (Beckwith \& Sargent 1996), labeled by Kant an "Urnebel."

The framework for understanding stellar formation is based on a rapidly evolving theoretical and observational picture. Although the formation of circumstellar disks has

\footnotetext{
${ }^{1}$ School of Physics, University of Sydney, Sydney NSW 2006, Australia; gekko@physics.usyd.edu.au.

${ }^{2}$ Smithsonian Astrophysical Observatory, MS 42, 60 Garden Street, Cambridge, MA 02138; jmonnier@cfa.harvard.edu.

${ }^{3}$ NASA Goddard Space Flight Center, Infrared Astrophysics, Code 685, Greenbelt, MD 20771; wcd@snoopy.gsfc.nasa.gov.

${ }^{4}$ Space Sciences Laboratory, University of California, Berkeley, CA 94720-7450; david@isi.mtwilson.edu,cht@ssl.berkeley.edu.
}

long been favored, for the case of massive stars there have been historical problems with getting models that fit the shape of the spectral energy distribution (SED) (Hillenbrand et al. 1992) to account for the luminosities in the near-IR (Hartmann, Kenyon, \& Calvet 1993) and with forbidden line profiles not matching expectations (Böhm \& Catala 1994). It was also found that the SED could be fitted by spherically symmetric shells (Miroshnichenko, Ivezić, \& Elitzur 1997; Pezzuto, Strafella, \& Lorenzetti 1997) or by composite shell-disk models (Miroshnichenko et al. 1999).

Recent observational evidence has argued both for and against the presence of disks. Millimeter-wave interferometry has found evidence of large-scale rotation in Herbig Ae stars (Mannings \& Sargent 1997, 2000), albeit at much larger spatial scales than the expected sizes of the inner disks. However, similar studies of Herbig Be stars (Fuente et al. 2001) conclude that the disks have dissipated at an early stage, before the star becomes visible. Even restricting attention to just Herbig Ae stars, Hubble Space Telescope coronagraphic observations of AB Aur (Grady et al. 1999) show a more circularly symmetric structure, consistent with a disk viewed pole-on, contrasting with the millimeter observations.

Recent high-resolution imaging in the infrared has also been equivocal. Classical accretion disks have generally performed poorly in attempting to fit observations from the latest generation of long-baseline interferometers (MillanGabet et al. 1999; Millan-Gabet, Schloerb, \& Traub 2001; Akeson et al. 2000). More directly, the expected source asymmetries from a population of disks, most of which must be inclined to the line of sight, were not indicated Millan-Gabet et al. (2001). However, of the three systems for which the hot inner regions have been well resolved by full interferometric imaging in the infrared, HK Tauri B (Koresko 1998), LkH $\alpha 101$ (Tuthill, Monnier, \& Danchi 
2001), and MWC 349 (Danchi, Tuthill, \& Monnier 2001), clear disk morphologies (edge-on, face-on, and edge-on, respectively) have been established. In contrast to the nearIR, in which disks have proven larger than expected, Hinz, Hoffmann, \& Hora (2001) failed to detect extended flux around a sample of Herbig Ae stars from mid-IR nulling interferometry.

Modelers are now rethinking the simple thin/flared-disk geometries with power-law thermal profiles, now seen by many as inadequate in light of the measurements of MillanGabet and others, and promising new candidates for reconciling some of the discrepancies are emerging. One new model (Dullemond, Dominik, \& Natta 2001) involves an inner circumstellar cavity whose radius is set by thermal dust evaporation that adjoins a flaring disk geometry (Chiang \& Goldreich 1997) at larger radii. Such flared disks may have thermal instabilities causing runaway local heating, puffed walls, and self-shadowing of more distant regions (Dullemond 2000). The resulting geometry is capable of intercepting more of the stellar radiation, thus boosting the near-IR excess while maintaining excellent fits to the SED. This has been verified experimentally with new spectral data on four young stars (Natta et al. 2001).

$\mathrm{LkH} \alpha 101$ was first identified as the source of illumination of the irregular reflection nebula NGC 1579 by Herbig (1956), who matched the $\mathrm{H} \alpha$ spectrum with a faint, deeply embedded star lying $\sim 5^{\prime \prime}$ within the border of a dark lane crossing the few arcminute-sized nebula. In the years since this discovery, it has become one of the most studied young stellar objects, with observations spanning the spectrum. Despite this, pinning down many of its basic properties has proved elusive. The $800 \mathrm{pc}$ distance established by Herbig (1971) was based on measurement of two nearby stars believed to be in association. Recently, Stine \& O'Neal (1998) made a strong case, based on radio photometry, that the system is much closer, suggesting instead that it is located in an extension of the Taurus-Auriga star formation complex at $160 \mathrm{pc}$.

Part of the difficulty has been the complexity of the highly anisotropic circumstellar environment, with at least four molecular clouds (Redman et al. 1986; Barsony et al. 1990) surrounding $\mathrm{LkH} \alpha 101$ and its associated $\mathrm{H}$ II region, S222 (Herbig 1956). A deeply embedded population of up to hundreds of probable protostars, pre-main-sequence low-mass stars, and brown dwarf candidates has been detected in the radio (Becker \& White 1988; Stine \& O'Neal 1998) and infrared (Barsony, Schombert, \& Kis-Halas 1991; Aspin \& Barsony 1994). Estimates of the visible extinction vary wildly in the literature by almost $10 \mathrm{mag}$, between $A_{v}=9.4$ (Barsony et al. 1990) and 18.5 mag (Hou, Jiang, \& Fu 1997).

This paper presents results from extremely high angular resolution imaging obtained with interferometric techniques. This has allowed observation of the hot, young, selfluminous disk surrounding $\mathrm{LkH} \alpha 101$ in the near-IR and mid-IR. The observational methods and the two different instruments used to obtain the data are described in $\S 2$, with the results given in $\S 3$. The astrophysical interpretations are discussed in $\S 4$, while $\S 5$ contains a summary of the important findings.

\section{OBSERVATIONS}

Observations were made at a number of epochs over the interval 1997-2001 and utilized two separate instruments to secure high-resolution imaging data in the near-IR and midIR. Near-IR observations were taken with the Keck I telescope, while the mid-IR interferometry was performed with the UC Berkeley Infrared Spatial Interferometer (ISI). These are briefly described in turn below.

\subsection{Near-IR Interferometry}

Observations at the Keck I telescope employed the technique of aperture masking interferometry, in order to recover information out to the diffraction limit (Tuthill et al. 2000 ) of the $10 \mathrm{~m}$ primary mirror. Placing a mask over the telescope pupil has been shown under some circumstances to confer signal-to-noise ratio advantages over full-pupil speckle interferometry (Haniff \& Buscher 1992). A large number of rapid-exposure, high-magnification data frames were processed to extract Fourier amplitudes and closure phases, enabling images to be produced from a self-calibration algorithm based on the maximum entropy method (MEM; Sivia 1987; Skilling \& Bryan 1984). A detailed description of the apparatus and data processing can be found in Tuthill et al. (2000), and its application in the study of young stars is demonstrated in Bittar et al. (2001) and Tuthill et al. (2001).

An observing log of near-IR observations is given in Table 1, showing the dates, filters, and point-source calibrator stars used for each data set. The observing wavelength was selected from the standard complement of interference filters in the Near-IR Camera (NIRC) facility, which offered a range of bandwidths (from about $1 \%$ to $20 \%$ ) over the near-IR region (Matthews et al. 1996). The characteristics of filters used are given in Table 2. All observations utilized the annular ring-shaped pupil (Tuthill et al. 2000), with the exception of the 1999 February 5 observation within the $J$ band, which utilized the full unobstructed pupil because of the relatively low apparent luminosity of $\mathrm{LkH} \alpha 101$ in this spectral region. An additional difficulty with the $J$ - and some $H$-band observations was associated with the fact that

TABLE 1

Journal of Near-IR Keck ObServations

\begin{tabular}{|c|c|c|}
\hline $\begin{array}{l}\text { Date } \\
\text { (UT) }\end{array}$ & Filter & Calibrator Star \\
\hline \multirow[t]{3}{*}{1997 Dec $17 \ldots .}$. & $H$ & HD 27349 \\
\hline & KCONT & HD 27349 \\
\hline & PAHCS & HD 27349 \\
\hline \multirow[t]{3}{*}{1997 Dec $19 . . .}$. & $H$ & HD 27349 \\
\hline & KCONT & HD 27349 \\
\hline & PAHCS & HD 27349 \\
\hline \multirow[t]{4}{*}{1998 Sep $30 \ldots .}$. & $H$ & 54 Per \\
\hline & $\mathrm{Br} \gamma$ & HD 27349 \\
\hline & $\mathrm{CH} 4$ & HD 27349 \\
\hline & PAHCS & HD 27349 \\
\hline \multirow{2}{*}{1999 Jan 6 ........ } & $H$ & 54 Per \\
\hline & $\mathrm{CH} 4$ & 54 Per \\
\hline 1999 Feb $5 \ldots \ldots .$. & $J$ & HD 28423 \\
\hline \multirow[t]{2}{*}{2000 Jan $26 \ldots}$. & $H$ & 54 Per \\
\hline & $\mathrm{CH} 4$ & 54 Per \\
\hline \multirow[t]{2}{*}{2001 Jul $30 \ldots \ldots$} & $H$ & HD 27349 \\
\hline & $\mathrm{CH} 4$ & HD 27349 \\
\hline \multirow[t]{4}{*}{2001 Sep 4 ........ } & $J$ & HD 28423 \\
\hline & $H$ & 54 Per \\
\hline & $\mathrm{CH} 4$ & HD 27349 \\
\hline & $K$ & HD 27349 \\
\hline
\end{tabular}


TABLE 2

Interference Filters Used in THE NEAR-IR

\begin{tabular}{|c|c|c|}
\hline Name & $\begin{array}{c}\lambda_{0} \\
(\mu \mathrm{m})\end{array}$ & $\begin{array}{c}\delta \lambda \\
(\mu \mathrm{m})\end{array}$ \\
\hline$J \ldots$ & 1.251 & 0.292 \\
\hline$H$ & 1.658 & 0.333 \\
\hline $\operatorname{Br} \gamma \ldots \ldots \ldots \ldots$ & 2.165 & 0.022 \\
\hline К & 2.214 & 0.427 \\
\hline KCONT .... & 2.260 & 0.053 \\
\hline $\mathrm{CH} 4 \ldots \ldots \ldots . . . .$. & 2.269 & 0.155 \\
\hline PAHCS....... & 3.083 & 0.101 \\
\hline
\end{tabular}

the finest fringes were beyond the Nyquist sampling limit of the camera. This prevented recovery of data at a few of the very longest $H$-band baselines, while for the $J$ band even intermediate baselines had serious problems with fringe power aliasing, reducing the effective resolution to that of a $4 \mathrm{~m}$ telescope.

\subsection{Mid-IR Interferometry}

Mid-IR visibility data were obtained at $11.15 \mu \mathrm{m}$ with the ISI, a two-element heterodyne stellar interferometer located on Mount Wilson in California. Both telescopes are mounted within movable semitrailers which allowed reconfiguration of the baseline over the course of these measurements. Periodic observations of $\mathrm{K}$ giant stars $\alpha$ Tau and $\alpha$ Boo were utilized to calibrate fringe visibilities and to monitor drifts to within a few percent accuracy. Detailed descriptions of the apparatus, observing, and data reduction procedures can be found in Hale et al. (2000). A journal of $\mathrm{LkH} \alpha 101$ observations taken with the ISI is provided in Table 3. Spatial frequencies and position angles listed are averages of a small range spanned during the course of observations.

\section{RESULTS}

\subsection{Multiwavelength Near-IR Images}

Multiwavelength images of $\mathrm{LkH} \alpha 101$ are given in Figure 1 . For the $J$ - and $K$-band images, a single map produced from data taken in 2001 September is shown. The remaining images are averages of four separate observations over the interval 1997 December-1999 January, with a stronger weighting given to high signal-to-noise ratio data obtained when observing conditions were favorable. Although this averaging of maps could help reduce random noise, care had to be taken not to include data from widely separate epochs, as secular changes in appearance have been detected in this source, as described in more detail below.
The main features presented by this system are a bright, resolved, circular disk and a separate unresolved companion about 180 mas to the east-northeast. The resolved component exhibits a central hole, or depression in flux, and a marked asymmetric brightening toward the west-southwest limb. These basic features were reliably reproduced over multiple epochs under a range of different experimental conditions.

It is important, however, to point out inherent imperfections in this imaging process. Spurious structures are present in the images, for example, the faint circular ring appearing at the lowest contour level in the $K, \mathrm{KCONT}$, and $\mathrm{CH} 4$ maps, at a radius of around 90 mas. This feature would be familiar to radio astronomers as a "grating ring" and is caused by incomplete Fourier sampling. It should also be emphasized that the images are reconstructions, based on the maximum entropy technique, of a target that is not highly resolved. There is a very large parameter space of images that would fit to the data; the maximum entropy solution attempts to choose (in some sense) the smoothest, most featureless map from this set (see, e.g., Skilling \& Bryan 1984; Narayan \& Nityananda 1986). This implicit assumption is not always the best one to make in the context of astronomical imaging; however, for the present we adopt it as the standard starting point. The implications of using alternate initial assumptions are explored in $\S 4.4$.

When comparing maps made in different colors, it is important to be mindful of changes in the performance of the imaging system. Moving to longer wavelengths results in the loss of system resolution. This is clearly demonstrated in the $3 \mu \mathrm{m}$ (PAHCS) image, which is consistent with having the same basic morphology seen in the $2 \mu$ m images; however, the lower resolution is not capable of revealing the fine detail.

The images at shorter wavelengths in the $J$ and $H$ filters should, on the other hand, have higher resolution. Unfortunately, this theoretical improvement is not realized in the maps we see. The main reasons for this are (1) the negative impact of the seeing becomes greater for shorter wavelengths; (2) the pixel scale of the NIRC camera is not adequate to properly sample the finest interference fringes in this wavelength region; and (3) the target is relatively dim, forcing the use of wide-bandwidth filters, making calibration more difficult (Tuthill et al. 2000). In the case of the $J$ filter, no mask was used at all, resulting in further noise from atmospheric turbulence. These factors combine to result in a higher level of noise in the reconstructed images and a consequent loss of detail as compared with the $K$-band maps.

The set of $2 \mu \mathrm{m}$ images ( $K, \mathrm{KCONT}$, and $\mathrm{CH} 4)$ in Figure 1 presents the opportunity to examine whether the bandwidth of the observation is a factor in determining the appearance of the image. These filters have similar center

TABLE 3

JOURNAL OF MID-IR ISI OBSERVATIONS

\begin{tabular}{ccccc}
\hline \hline Date & $\begin{array}{c}\text { Telescope Separation } \\
(\mathrm{m})\end{array}$ & $\begin{array}{c}\text { Spatial Frequency } \\
\left(10^{5} \mathrm{rad}^{-1}\right)\end{array}$ & $\begin{array}{c}\text { Position Angle } \\
(\mathrm{deg})\end{array}$ & Visibility \\
\hline 1998 Oct.... & 4.0 & 3.28 & 73 & $0.964 \pm 0.087$ \\
1999 Sep..... & 13.3 & 11.47 & 106 & $0.648 \pm 0.068$ \\
\hline
\end{tabular}

a Position angle is measured in degrees east of north. 


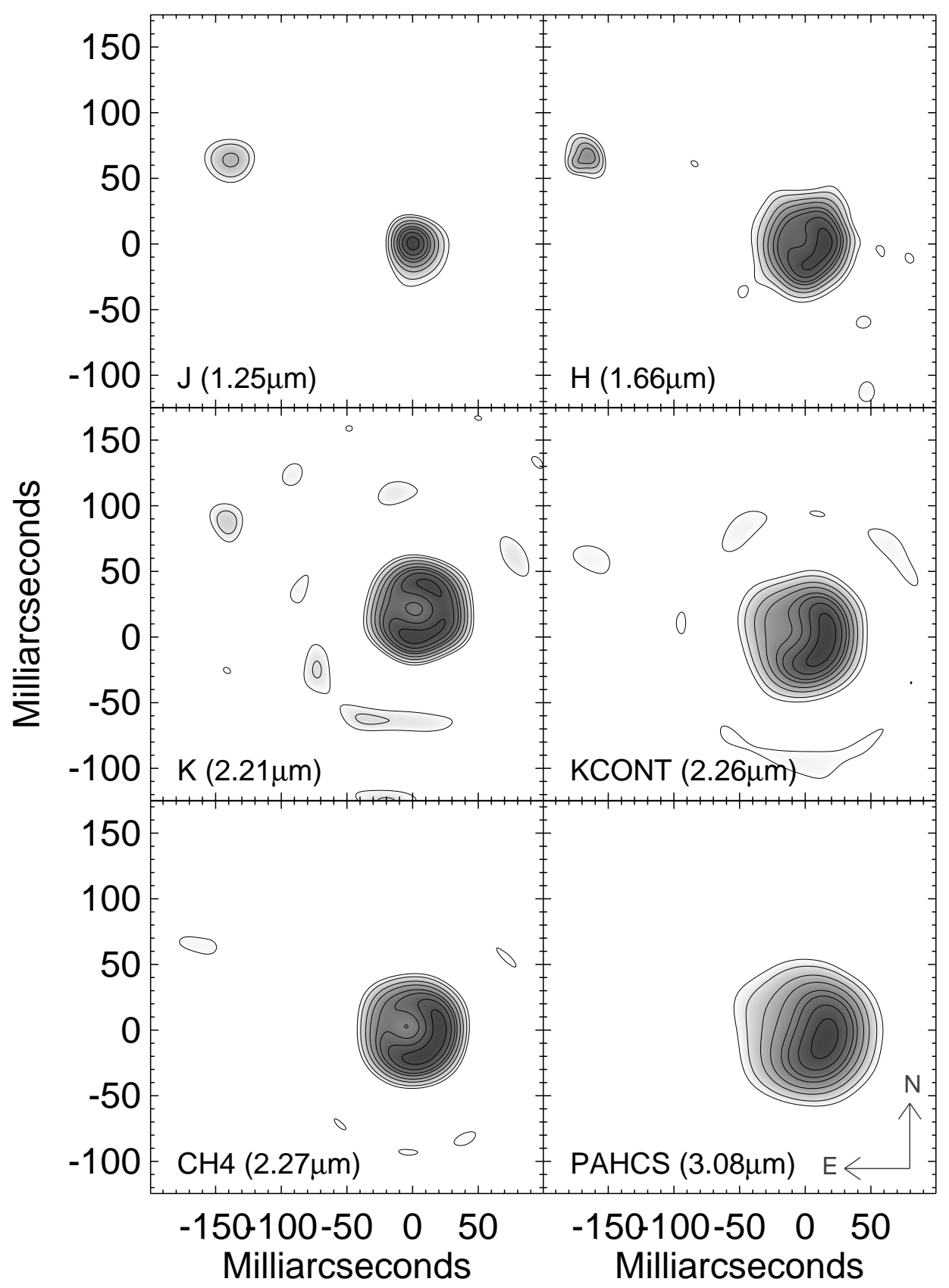

Contours (\% of Peak): 1251020305080

FIG. 1.-Images of $\mathrm{LkH} \alpha 101$ reconstructed from aperture masking interferometry data taken over six different filter bandpasses. To enhance the signal-tonoise ratio, most frames depicted are the average of a number of individual reconstructions taken at various epochs, as described in the text.

wavelengths (see Table 2); however, the fractional bandpasses are approximately $20 \%, 7 \%$, and $2 \%$ for $K, \mathrm{CH} 4$, and KCONT, respectively. Some apparent differences were found to be due to changes with observing epoch (see $\S 3.3$ below), as the $K$-filter image of Figure 1 differs by a couple of years from the others. A more detailed quantitative examination, comparing only images from the same epochs, revealed no systematic differences with filter bandwidth. Indeed, this should not be surprising, as there are no strong spectral features that would imply a different origin for light isolated by these three filters.

\subsection{Angular Sizes and Simple Brightness Models}

Although the images given in Figure 1 do give a good representation of the structure of $\mathrm{LkH} \alpha 101$, it is desirable to take a step backward toward the raw observables in extracting quantitative information. The prime reason for this is that fitting model brightness distributions to the observed visibilities and closure phases is a far more direct process than measuring intensities and sizes from an image reconstructed by a complex nonlinear algorithm.

Furthermore, because of limited Fourier coverage, the mid-IR interferometry from the ISI could not be inverted to produce a map for comparison with those in Figure 1. Information on the substantially cooler material to which the ISI is sensitive needs to be incorporated through a process of fitting simple model brightness profiles to the data.

The first set of fits made were to extract the position and relative flux of the $\sim 180$ mas binary companion in the nearIR data. The fitting algorithm employed both gradient 
TABLE 4

Simple Model Fits to Visibility Data

\begin{tabular}{|c|c|c|c|c|c|}
\hline \multirow[b]{2}{*}{$\begin{array}{c}\text { WAVELENGTH } \\
(\mu \mathrm{m}) \\
(1)\end{array}$} & \multirow[b]{2}{*}{$\begin{array}{l}\text { BINARY COMPANION } \\
\text { RELATIVE Flux } \\
\text { (2) }\end{array}$} & \multirow{2}{*}{$\begin{array}{l}\text { GAUSSIAN DISK } \\
\text { FWHM } \\
\text { (mas) } \\
(3)\end{array}$} & \multicolumn{3}{|c|}{ Disk + Point Source } \\
\hline & & & $\begin{array}{l}\text { Disk Flux } \\
\text { (4) }\end{array}$ & $\begin{array}{l}\text { Disk FWHM } \\
\text { (mas) } \\
\text { (5) }\end{array}$ & $\begin{array}{l}\text { Point Flux } \\
\text { (6) }\end{array}$ \\
\hline 1.236. & $0.140 \pm 0.021$ & $16.1 \pm 0.8$ & $0.31 \pm 0.03$ & $36.8 \pm 3.7$ & $0.55 \pm 0.03$ \\
\hline $1.658 \ldots \ldots \ldots \ldots . .$. & $0.040 \pm 0.013$ & $36.4 \pm 0.8$ & $0.84 \pm 0.02$ & $42.1 \pm 2.4$ & $0.12 \pm 0.02$ \\
\hline $2.265 \ldots \ldots \ldots \ldots \ldots$ & $0.008 \pm 0.003$ & $43.7 \pm 1.8$ & $\ldots$ & $\ldots$ & $\ldots$ \\
\hline $3.083 \ldots \ldots \ldots \ldots \ldots . . . . . .$. & $\ldots$ & $45.7 \pm 1.0$ & $\ldots$ & $\ldots$ & $\ldots$ \\
\hline $11.15 \ldots \ldots \ldots \ldots \ldots$ & $\ldots$ & $62.6 \pm 10.9$ & $\ldots$ & $\ldots$ & $\ldots$ \\
\hline
\end{tabular}

Notes. - Col. (2) gives the fractional flux of the binary companion, while Col. (3) gives the best-fitting FWHM of a Gaussian disk model of the central resolved component. Cols. (4)-(6) give fits of a more complex two-component model to the $J$ - and $H$-filter data only, as described in the text. All errors were computed by observing the statistical spread in values found by fitting the relevant models to separate, independently recorded data sets spanning a number of epochs.

descent and grid searching to find the optimum $\chi^{2}$ binary parameters from the calibrated visibility and closure phase data. Relative fluxes of the companion are given in column (2) of Table 4 for wavelengths in the $K$ band and shorter (the companion was not detected at long wavelengths), while separations are discussed in $\S 3.3$. The companion can be seen to be very much bluer than the resolved disk component, with its relative flux rising rapidly from the $K$ band through to the $J$ band.

Having established the parameters of the binary companion, it was possible to artificially remove it from the Keck data. This was done using the simplistic approximation of a binary with two point components; however, this proved easily adequate for the purpose of isolating the visibility function of the bright resolved disk. Two-dimensional visibility data were then averaged azimuthally to enhance the signal-to-noise ratio.

Data at four wavelengths through the near-IR (Keck data) and at $11.15 \mu \mathrm{m}$ (ISI data) are shown in Figure 2. Measurements from the entire course of the observing campaign are used, with a higher weighting given to the best epochs. Visibilities were fitted using simple circular Gaussian model intensity profiles, with the FWHM of the best fit given in column (3) of Table 4. Although the images of Figure 1 show that the intensity profile of $\mathrm{LkH} \alpha 101$ is better represented by a disk with a central depression than a Gaussian, we prefer here a simple one-parameter model, to give a robust estimator of the overall size. Despite its simplicity, the fits of this simple model in Figure 2 are quite good.

The most important thing to notice from the Gaussian disk fits of Figure 2 is the dramatically smaller size of the central disk in the $J$ band. According to the FWHM sizes given in Table 4, $\mathrm{LkH} \alpha 101$ shows a steady, slow decrease in size over the large wavelength interval from 11.15 to 1.658 $\mu \mathrm{m}$, then a sudden halving between 1.658 and $1.236 \mu \mathrm{m}$. Indeed, this dramatic reduction is also evident in Figure 1, which shows the $J$-filter image having a barely resolved core compared with images at other wavelengths.

Some increase in size with wavelength is to be expected from thermal considerations: cooler dust farther out will give a greater fractional contribution at long wavelengths. This probably explains the $(\sim 20 \%)$ increase from 1.658 to $3.083 \mu \mathrm{m}$ and the ( $27 \%)$ increase from 3.083 to the ISI data point at $11.15 \mu \mathrm{m}$. Although thermal radiative transfer modeling is required to fully exploit the implications of these data for the structure of $\mathrm{LkH} \alpha 101$, the modest nature of these increases seems to point to a fairly compact, bounded structure, whose size is therefore not a strong function of observing wavelength.

The most logical explanation for the sudden drop in apparent size from 1.658 and $1.236 \mu \mathrm{m}$ is that the central star itself is beginning to outshine the disk at shorter wave-

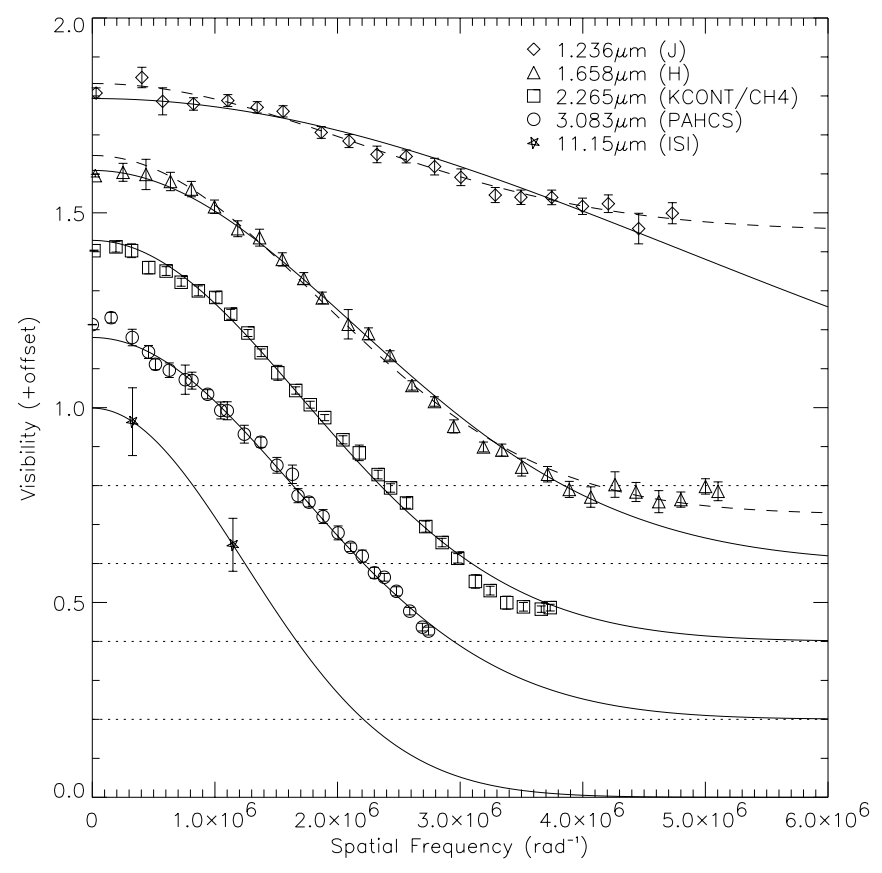

FIG. 2.-Visibility curves of $\mathrm{LkH} \alpha 101$ from Keck aperture masking and ISI interferometry data. Keck data have had successive visibility offsets of 0.2 added (horizontal dotted lines), in order to separate the curves on the page. The $1.236,1.658$, and $2.265 \mu \mathrm{m}$ curves have had excursions because of the presence of the binary companion removed (see text). To enhance the signal-to-noise ratio for the Keck data, curves depicted are the average of a number (typically 3-5) of separate observations taken at various epochs. Overplotted solid lines show a best-fitting circular Gaussian function to the data at each wavelength. For the $J$ - and $H$-filter observations, a more complicated, two-component model is also shown (dashed line) that is discussed in the text. 
lengths. Visibility and closure phase data severely limit the range of allowed locations of this stellar component to near the center of the resolved disk. Placed anywhere else, it would generate strong excursions, which were not observed.

Further strong evidence of the presence of a hot (blue) point source comes from examination of the $H$-filter visibility curve of Figure 2. At high spatial frequencies (above $\sim 4 \times 10^{6} \mathrm{rad}^{-1}$ ) the measured data depart systematically from the Gaussian disk fit, in a manner consistent with the presence of a weak point source. This is illustrated with the dashed curve, showing the unresolved component holding up the visibilities at high spatial frequency.

Table 4 shows results from fitting two-component " disk + point source" models consisting of a point component embedded in a Gaussian disk. (Technically, these might be regarded as three-component models: recall that the binary companion has already been subtracted from these data.) This model was found to reduce the $\chi^{2}$ misfit to the $H$-band visibility data by an average factor of 1.9 over the seven epochs recorded, although this improvement comes at the expense of an extra free model parameter.

The preferred model for $\mathrm{LkH} \alpha 101$ can therefore be summarized as a resolved disk (approximated as a Gaussian of around 40 mas FWHM) showing only a modest increase in apparent size across the entire near-IR, together with a distant blue companion (contributing $14 \%$ at $J$ ) and a bright, embedded blue point source (contributing 55\% at $J$ ). Before proceeding with this interpretation, however, it is important to note that alternate scenarios might be indicated, particularly with the benefit of full radiative transfer modeling and including more complex effects, such as scattering, which may also play a role in altering the appearance of the disk toward the blue.

In a recent imaging study of young massive stars, Leinert et al. (2001) reported the presence of an extended (0".9) halo contributing $15 \%$ of the flux in $H$ band. Evidence of this was not seen in the visibility curves of Figure 2, where a sharp drop would be expected over the nearest few points from the origin. Interferometric techniques (including both the results here and those of Leinert et al. 2001) can prove unreliable for the very shortest baselines (the so-called seeing spike problem; see, e.g., Tuthill et al. 2000). However, despite this, a halo as strong as $15 \%$ would give signals that lie well above this noise process and should have been seen here. We hope that discrepancies such as this are sorted out as the techniques mature.

\subsection{Time Evolution of Images}

With observations spanning almost 4 years, careful comparisons were made in order to determine if $\mathrm{LkH} \alpha 101$ exhibited significant changes of morphology with time. In particular, the location of the binary companion was carefully monitored for evidence of orbital motion. A plot of the separation (in right ascension and declination) is given in Figure 3. The error bars are larger than can be obtained for simpler binaries consisting of two point-source components because of ambiguity introduced by the need to locate a fiducial fixed point within the complex disk structure. For this purpose, the center of the circular disk (ignoring the asymmetric limb brightening) was chosen.

Figure 3 shows the companion to be moving toward the west, with almost all the change in the R.A. coordinate. Fitting a uniform velocity to the data yields a motion of

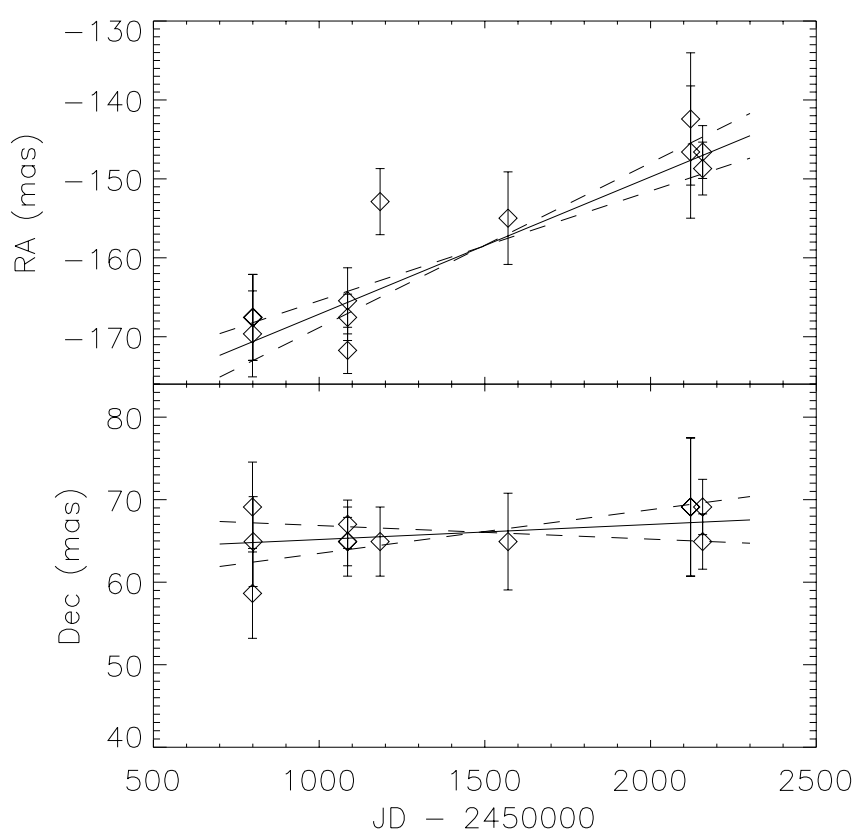

FIG. 3.-Relative position of $\mathrm{LkH} \alpha$ 101's companion as a function of Julian Date. Top: Right ascension; bottom: declination. The best-fit uniform-velocity model (solid line) is overplotted with the $1 \sigma$ error in the component velocities indicated (dashed lines).

$6.4 \pm 1.3$ mas $\mathrm{yr}^{-1}$ at a position angle of $275^{\circ} \pm 12^{\circ}$, with a starting location of $182.5 \pm 2.4$ mas separation and a position angle of $69^{\circ} \pm 2^{\circ}$ on 1997 December 17 (JD 2,450,799). Data are neither extensive nor precise enough to justify fitting of a full orbit at this stage. The motion can be directly visualized from examination of the images presented in Figure 1 . The $J$ and $K$ panels, whose data were taken in 2001 September, show the companion visibly farther to the west than the $H, \mathrm{KCONT}$, and $\mathrm{CH} 4$ images recorded in and around 1998.

Future monitoring of this companion to determine the orbit should help in pinning down the basic properties of the system, as discussed further in $\S 4$.2. It will also be interesting to determine if the companion orbit is coplanar with the disk, as this has implications for the mechanism of formation of the binary system. The interested reader is referred to the discussion in Koresko (1998), who found HK Tauri B to have a disk that was not coplanar with the binary companion.

Systematic changes of structure were also recorded for the resolved disk component of $\mathrm{LkH} \alpha 101$. This was studied by comparison of the highest-fidelity images, which were all produced in the $K$-band spectral region. Images were superior, as this represented the best compromise between higher theoretical resolution (shorter wavelengths), more benign atmospheric noise properties (longer wavelengths), and low contamination by the central point source (longer wavelengths). Figure 4 gives a series of $K$-band images from 1997 December through 2001 September depicting just the central disk component.

Although the basic structure of an asymmetrically limbbrightened circular disk with a central hole or depression is preserved in this series, there are more subtle changes associated with the peak intensity. It can be seen that the brightest quadrant, initially to the west-southwest $(1997 / 1998)$, gradually lengthens and shifts to the south $(1999 / 2000)$, before 


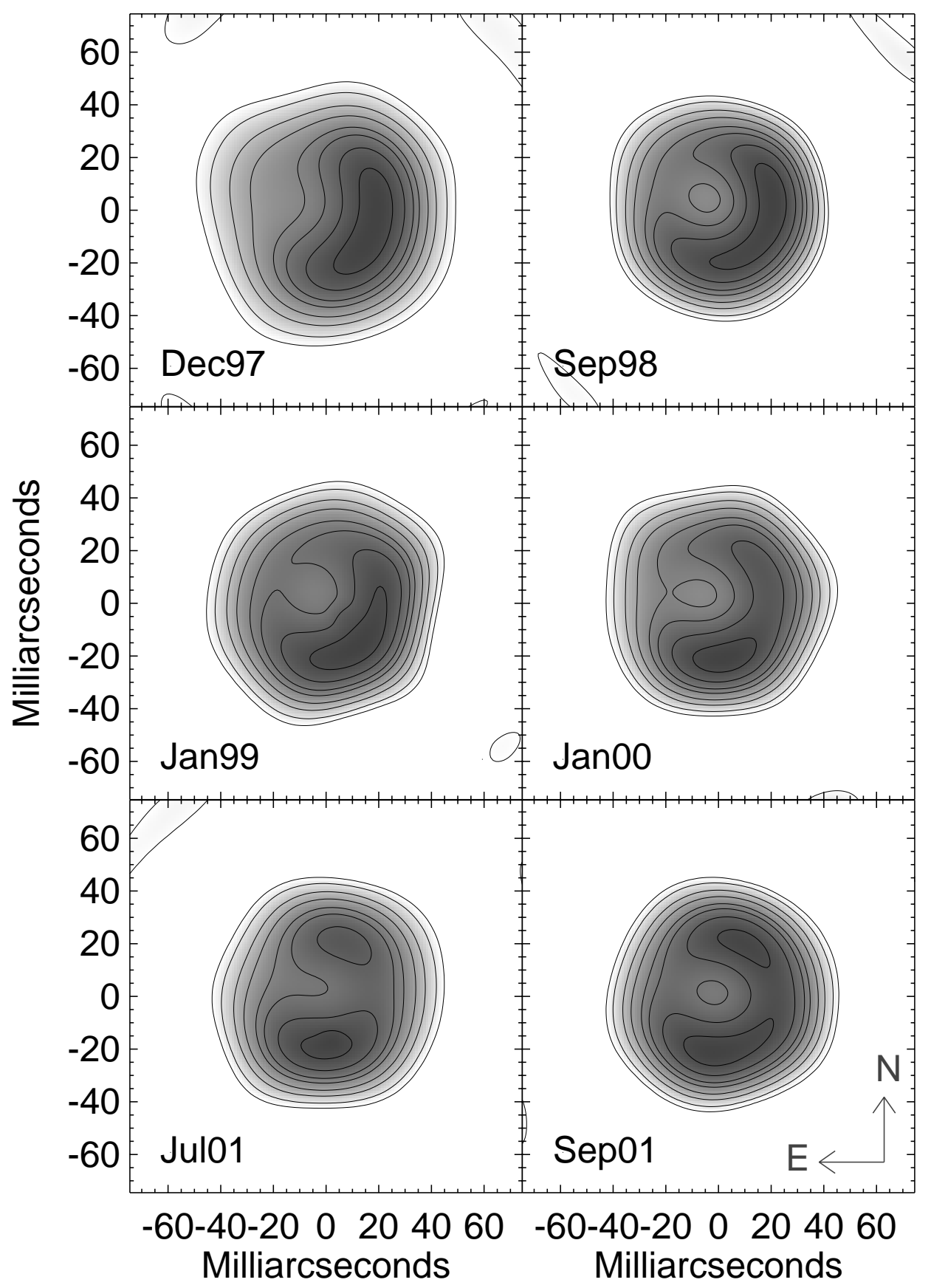

Contours (\% of Peak): 1251020305080

FIG. 4. $-K$-band images of the resolved disk of $\mathrm{LkH} \alpha 101$ from data taken at six different epochs. With the exception of the 1997 December observations, for which the KCONT filter was used, all observations were made with the $\mathrm{CH} 4$ filter.

finally splitting into two: a northern component and a slightly brighter southern component (2001). Changes of this kind might be expected from noisy data in a single map, but noisy data are unlikely to be a problem in this case, as these changes are highly systematic over time, and multiple contemporaneous observations at each individual epoch all corroborate the average map at that epoch to a high degree.

However, an important caveat on the interpretation of reconstructed structure near the diffraction limit needs to be reemphasized at this point. Although we believe that there has been real evolution of the morphology of $\mathrm{LkH} \alpha$ 101's resolved disk, the precise details of this change are unclear.
The maximum entropy picture of the north-south migration and splitting of what was formerly a bright crescent to the west is only one of many possible valid models. This question of ambiguity over the details of reconstructed images is discussed at greater length in $\S 4$ 4. However, despite the uncertainties, the changes in the raw visibilities and phases that cause the evolution evident in Figure 4 are real.

Photometry of $\mathrm{LkH} \alpha 101$ could also be extracted from the data, utilizing the unresolved calibration stars as photometric references. Although the experiment was clearly not optimized for such measurements, we were able to establish that the infrared fluxes were constant to within an uncer- 
tainty of $10 \%$ over the course of the observing campaign. Absolute flux levels were found to be in good agreement with published photometry discussed in $\S 4.3$.

\section{DISCUSSION}

\subsection{LkHo 101: a Face-on Accretion Disk System}

The first qualitative impression given by the appearance of the images of $\mathrm{LkH} \alpha 101$ is that they conform well to our preconceptions for a young system surrounded by an accretion disk. The interpretation of the circular outline of the dust as a face-on view onto a disk, rather than as a spherical structure, is well justified. The inner regions have long been known to be highly inhomogeneous, as testified by the bright reflection nebula NGC 1579, which must have a low line-of-sight opacity to the central star. Further verification comes from studies of line widths and excitation conditions (Simon \& Cassar 1984), revealing the existence of a number of regions. A polar viewing angle for this star has also been favored in the literature (Barsony et al. 1990). The degree of circularity was measured by fitting to the images with the best signal-to-noise ratios and ignoring the brightest (asymmetric) structures. No systematic elongations were found in any direction, enabling a limit of $\lesssim 35^{\circ}$ to be placed on any tilt of the polar axis from the line of sight (assuming the disk itself to be circular and flat).

The marked central depression, or gap, evident in the images of Figures 1 and 4 points to a hole, or at least an optically thin region at the center of the disk surrounding the central star. Such central cavities have long been invoked in order to help with the fitting of the broadband spectra, and they are also found in more recent models (Hollenbach et al. 1994; Yorke \& Welz 1996). The latter scenarios apply to massive stars upon attainment of their mainsequence luminosity, when they commence the erosion of their accretion disks in $\lesssim 10^{6} \mathrm{yr}$ with radiation and wind. It is possible that our images of $\mathrm{LkH} \alpha 101$ give the first direct view of this process at an early stage, with the star caught in this ephemeral phase, having etched away the inner disk to produce the dark hole at the center, but with substantial material still orbiting in a hot, close ring.

The final aspect of the image morphology that proves to be qualitatively instructive is the asymmetric, crescentshaped brightening to the southwest, best illustrated in Figure 4. The simplest interpretation is that the plane of the disk is tilted away from our line of sight in the southwest, allowing a clearer view to the hottest material on the inner wall facing the star in this quadrant. Conversely, in the northeast side of the disk, being closer to us, cooler material farther out is obscuring the hot inner parts. Such geometrical arguments are only consistent with a thick disk, torus, or strongly flared structure. Thin, flat disks, often encountered in the literature, will not produce such effects.

The details of the appearance of a photoevaporating disk, including a "peculiar horseshoe-like feature" for models with an inclination of $30^{\circ}$, were predicted in simulations of Kessel, Yorke, \& Richling (1998). A model $12 \mu$ m intensity map extracted from their paper is given in Figure 5, showing a morphology strikingly similar to our images of $\mathrm{LkH} \alpha 101$.

The figure was included to illustrate the way in which a somewhat inclined, centrally illuminated, thick torus can make a good match to our observations. Although such qualitative comparisons are highly encouraging, thick-disk

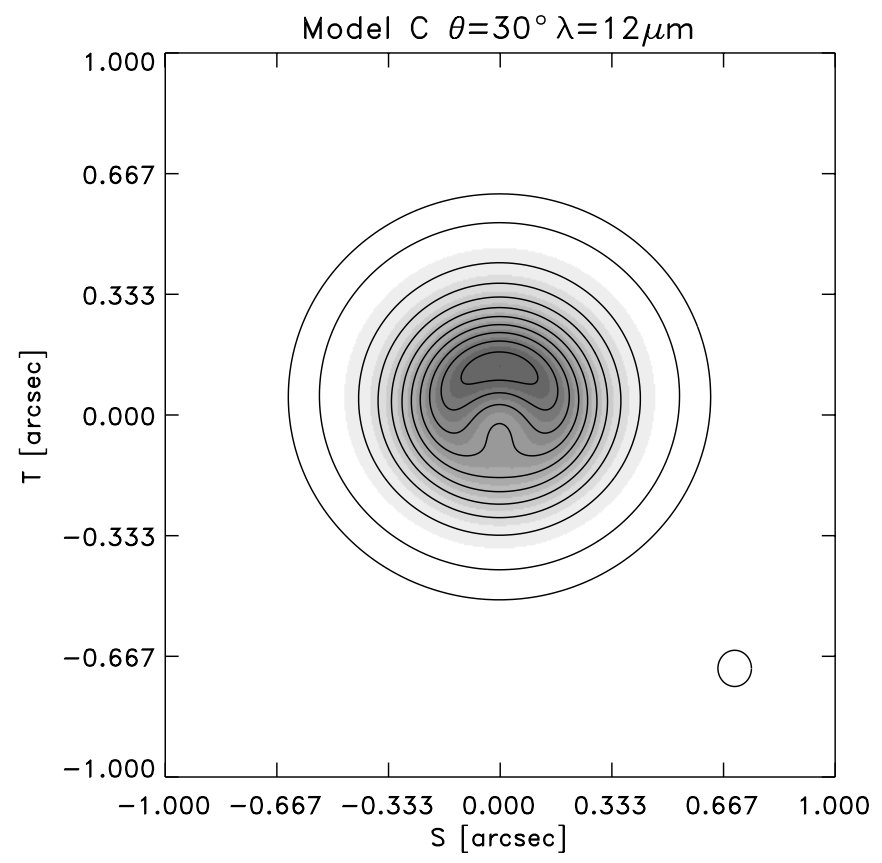

FIG. 5.-Model showing a simulated $12 \mu \mathrm{m}$ image of a photoevaporating disk viewed at an inclination of $30^{\circ}$, taken from Fig. $7 f$ of Kessel et al. (1998).

geometries with inner cavities are not unique to photoevaporating models, and establishing a physical model for this system requires more detailed quantitative analysis, as discussed below.

\subsection{Basic Properties}

Despite extensive observational study, there is a great deal of uncertainty over the basic properties of $\mathrm{LkH} \alpha 101$. A distance of $800 \mathrm{pc}$, established by Herbig (1971) from observations of nearby stars thought to be in association, had become entrenched in the literature. However, this was recently challenged by Stine \& O'Neal (1998), whose radio photometry pointed to a much closer distance, suggesting that the star may lie in an extension of the Taurus-Auriga star formation complex at $160 \mathrm{pc}$.

$\mathrm{LkH} \alpha$ 101's bolometric luminosity, estimated at $4.8 \times 10^{4} L_{\odot}$ (Barsony et al. 1990), was later revised downward after it was found that up to $75 \%$ of the flux might be coming from a deeply embedded cluster containing hundreds of objects (Barsony et al. 1991). If the $160 \mathrm{pc}$ distance scale is adopted, instead of $800 \mathrm{pc}$ assumed by these earlier authors, the resulting luminosity of $4.8 \times 10^{2} L_{\odot}$ would imply a mid-to-late B star. Although $\mathrm{LkH} \alpha 101$ may not be as highly luminous as the $\mathrm{O}$ star in earlier references, a midto-late B star now seems to be an underestimate. Radio observations showing an ionized shell (Harris 1976; Cohen, Bieging, \& Schwartz 1982), when corrected for a distance scale closer than 800 pc, find Lyman continuum photon fluxes, as would result from a significantly hotter early star (Panagia 1973). As the photoionizing flux is a strong function of spectral type, the radio photometry seems the most reliable indicator of the central star in this case.

Although revisions to the optical flux, such as an intermediate distance scale and greater relative contribution from $\mathrm{LkH} \alpha 101$ to the measured bolometric luminosity, might bring it more into accord with the radio data, there 
are many difficulties, such as known global anisotropies and uncertain optical depths, complicating the picture. However, we proceed with the assumption (based on matching radio photometry with expected Lyman continuum flux from given spectral types) that $\mathrm{LkH} \alpha 101$ is an early B0 B0.5 zero-age main-sequence (ZAMS) star, which implies an approximate mass $M_{*} \simeq 10-20 M_{\odot}$, an effective temperature $T_{\text {eff }} \simeq 25,000-30,000 \quad \mathrm{~K}, \quad$ a luminosity $L \simeq 10,000-25,000 L_{\odot}$, and a radius $R_{*} \simeq 5 R_{\odot}$ (Panagia 1973).

The measured properties of the binary (apparent separation 180 mas; motion $6.4 \pm 1.3$ mas yr $^{-1}$ ) from $\S 3.3$ allow us to set fully independent constraints on the distance. These are derived from the idea that the angular separation and velocity we see are related to the true parameters governing the binary motion (linear separation, semimajor axis, and orbital velocity) by the distance, combined with geometrical foreshortening factors. Although we know neither these geometrical factors nor the true mass, it can be seen below that estimates of distance can be discriminated by making simple probabilistic assumptions about these unknown quantities (for example, that we are not looking from an unlikely, highly foreshortened perspective at the binary).

First, if we take the $800 \mathrm{pc}$ distance scale, then the orbital period of a binary with semimajor axis 180 mas and an assumed system mass of $15 M_{\odot}$ is $446 \mathrm{yr}$. On the plane of the

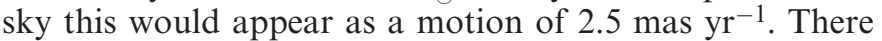
are a number of ways our measurement of 6.4 mas $\mathrm{yr}^{-1}$ could easily underestimate the true motion; however, it is more difficult to see how we have found a larger apparent motion. The enclosed system masses would need to be 100 $M_{\odot}$ in order to generate the observed apparent motion at this distance. The companion could be approaching periastron in a highly eccentric orbit and therefore moving faster than expected for its separation or, alternatively, the objects may not form a bound pair at all, in which case the apparent superposition we see will last $\lesssim 100 \mathrm{yr}$. Although they cannot be ruled out, none of these explanations seem very satisfactory, and we conclude $\mathrm{LkH} \alpha 101$ is likely closer than $800 \mathrm{pc}$.

If we consider now the $160 \mathrm{pc}$ distance, an apparent

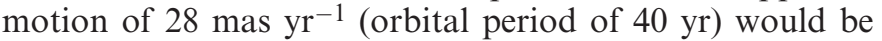
seen for a $15 M_{\odot}$ circular binary in the plane of the sky with radius 180 mas. As mentioned above, there are a number of ways in which our measurements could underestimate the true state of affairs. Apparent motions as low as 6.4 mas $\mathrm{yr}^{-1}$ could be due to a large, hidden, line-of-sight component to the velocity vector, although this is somewhat unlikely, as it requires close alignment of the present orbital direction to the line of sight. The separation of 180 mas used for the orbital computation might similarly be an underestimate due to foreshortening (the companion has a much larger separation, but on a vector close to our line of sight); however, this is statistically extremely unlikely, requiring much closer alignments. Allowing system masses as low as 5 and $10 M_{\odot}$ results in periods of 69 and $49 \mathrm{yr}$, giving apparent motions of 16 and 23 mas $\mathrm{yr}^{-1}$, respectively. The $5 M_{\text {。 }}$ assumption can match the observations without highly unlikely inclinations; however, it is doubtful that stars at this light end of this range are energetic enough to power the observed $\mathrm{H}$ II region. As before, it is also possible to fit the observations by assuming a highly eccentric orbit: in this case, the binary would need to have been observed moving slowly near apastron.
If we instead invert the question and ask what is the distance given the apparent motion, we arrive at $430 \mathrm{pc}$ for a 15 $M_{\odot}$ circular binary orbiting in the plane of the sky. However, we can do a little better than this, at least in a statistical sense, by making the assumption that we have observed one-dimensional projections of velocity and separation vectors taken from a random isotropic population (resulting in likely underestimation by a factor of $\pi / 4$ ). Accounting for this results in a likely distance of $\sim 340 \mathrm{pc}$, although values in the range 200-500 pc can be comfortably accommodated, given the uncertainties in the mass and projection angle and the experimental error on the apparent motion. Allowing for eccentricity widens this range still further, although numerical simulations fitting for various orbits did find far more solutions at the intermediate distance than at the extremes. Unfortunately, the $180 \mathrm{yr}$ period of such a system makes it unlikely that precise orbital elements, lifting some of the uncertainty in these calculations, can be derived soon.

In conclusion, although we can rule out neither of the earlier distance estimates, our imaging results favor an intermediate, $\sim 340 \mathrm{pc}$ scale. As the previous distance scales have not been established with any degree of certainty, we include this new value despite the large uncertainties in its derivation above. For the remainder of this paper, interpretations based on the short (Stine \& O'Neal 1998), intermediate (this work), and long (Herbig 1971) scales are given.

\subsection{Spectral Energy Distribution}

The observed SED for $\mathrm{LkH} \alpha 101$ is given in Figure 6, for which the data have been taken from various literature sources. The overplotted $750 \mathrm{~K}$ blackbody curve was found (Cohen \& Woolf 1971; Danen et al. 1995) to fit the near to mid-IR spectral regions. We were able to extract photometry from our own observations, and found the fluxes to be generally consistent with the literature values; in particular,

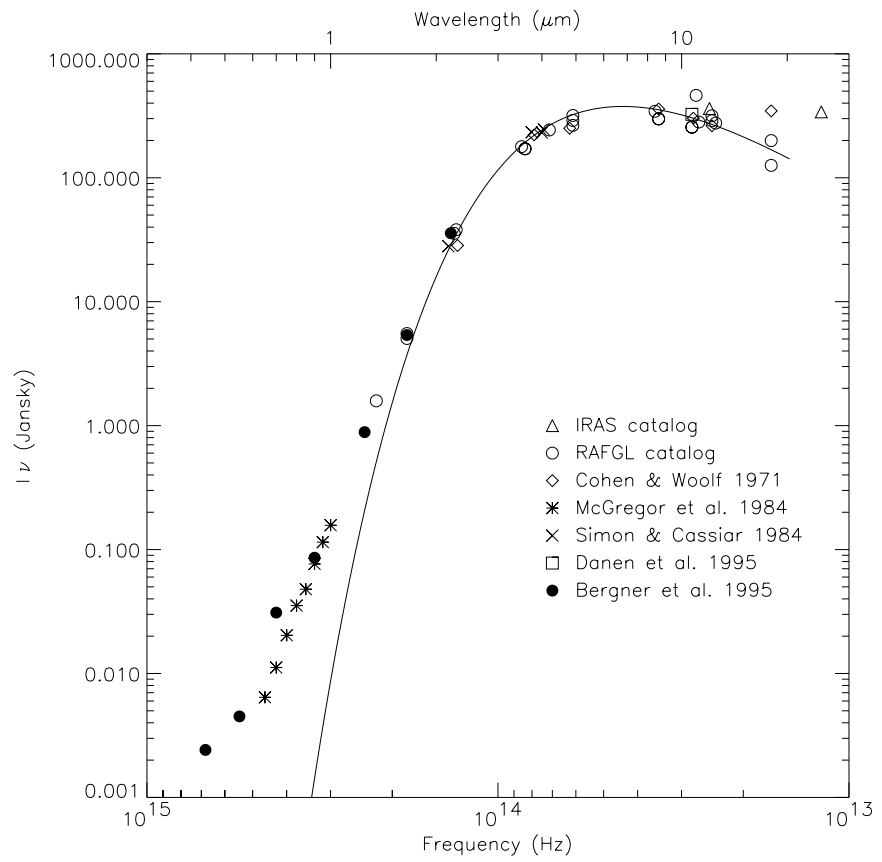

FIG. 6.-SED of LkH $\alpha 101$ from the optical through the mid-IR, taken from various literature sources, as indicated. The overplotted solid line is a 750 K blackbody curve (Cohen \& Woolf 1971; Danen, Gwinn, \& Bloemhof 1995). 
good agreement was found with the near-IR data of Bergner et al. (1995). We therefore presume that these historical data are fairly representative of the present epochs under study.

An analysis of the energetics of $\mathrm{LkH} \alpha 101$ and its associated region is complicated by the fact that it is known to lie behind thick clouds of obscuring material. The spectrum must therefore be corrected for the wavelength-dependent extinction (reddening). This has been done with dust constants appropriate for the so-called outer cloud dust (Mathis 1990) expected in regions of heavy obscuration.

Widely disparate estimates of the line-of-sight visual extinction $\left(A_{v}\right)$ can be found in the literature, with values of 9.4, 9.7, 11.2, 14.2, 15.7, and 18.5 obtained from, respectively, Barsony et al. (1990); McGregor, Persson, \& Cohen (1984); Kelly, Rieke, \& Campbell (1994); Thompson et al. (1977); Rudy et al. (1991); and Hou et al. (1997). In the following discussion, we proceed with the relatively recent, intermediate value of 11.2 from Kelly et al. (1994), although extinctions encompassing the entire range were trialed. When used to deredden the spectrum of $\mathrm{LkH} \alpha 101$, values to the upper end of the range were found to give unrealistic spectra, rising very sharply in the blue. At the other extreme (low $A_{v}$ ), the system could be modeled, but a relatively small, cool central star was found, making it difficult to reconcile with the radio measurements of Lyman continuum photon fluxes.

The dereddened spectrum of $\mathrm{LkH} \alpha 101$, showing only the visible and near-IR, is shown in Figure 7. The spectral data points (diamonds) were taken from Figure 6, averaging multiple measurements at the same wavelength when necessary. There is now a strong indication of a two-component curve to the SED, with a red spectrum dominating wavelengths longer than $K$ band, while visible regions appear to betray the presence of a hot blue object. The transition region between these two cases falls around $1 \mu \mathrm{m}$.

Utilizing information from the imaging and model fitting in $\S 3$, we are able to decompose the full SED of Figure 7 into separate component spectra, each corresponding to individual features identified using high-resolution techni- ques. First, we are able to extract the spectrum of the binary companion by noting that we have obtained measurements of its relative flux in the $J, H$, and $K$ bands, as given in Table 4. The resulting SED of the companion, isolated in this fashion, is plotted as triangular points.

In a similar fashion, the remaining flux from the total can be divided into components due to the disk and central point source; the flux ratios have again been taken from Table 4 . The central point source, originally identified in $\S 3.2$ from Figure 2, is indicated by stars in Figure 7, while the resolved component of the flux assumed to come from the disk is indicated by circles. Note that the central point component was only clearly identified at two wavelengths, $J$ and $H$, but we have also overplotted a central point component, which contributes $2.5 \%$ of the flux at $K$ band. Although there may be weak evidence of such a signal in the visibility curve of Figure 2, this was done mainly as an illustration of the expected $K$-band flux of the central component extrapolated from $J$ and $H$. For wavelengths longer than $K$, the circumstellar disk is expected to dominate the SED.

For each of the three components of the system identified, a blackbody curve has been plotted. Both stellar components have been modeled as having a temperature of 25,000 K (B0.5 ZAMS star; from Panagia 1973), although the limited spectral coverage and uncertainties on the photometry mean that this is not well constrained by the data. A nearIR dust temperature of $1000 \mathrm{~K}$ has been used to fit the resolved disk component, and in contrast to the stellar case, the temperature is fairly well constrained to lie within \pm 50 $\mathrm{K}$ of this value. The model SEDs for the central star, disk, and binary companion are shown in Figure 7 as dotted, dashed, and dot-dashed lines, respectively.

Constraining the angular sizes to match the recorded flux levels, we deduce that the dust shell must subtend an apparent area of $800 \mathrm{mas}^{2}$ on the sky (an area equivalent to a uniformly illuminated circle of radius 16 mas). This is in agreement, to within a factor of $\sim 2$, with the flux-weighted observed angular size of the dust cloud $\left(\sim 1500\right.$ mas $^{2}$; radius

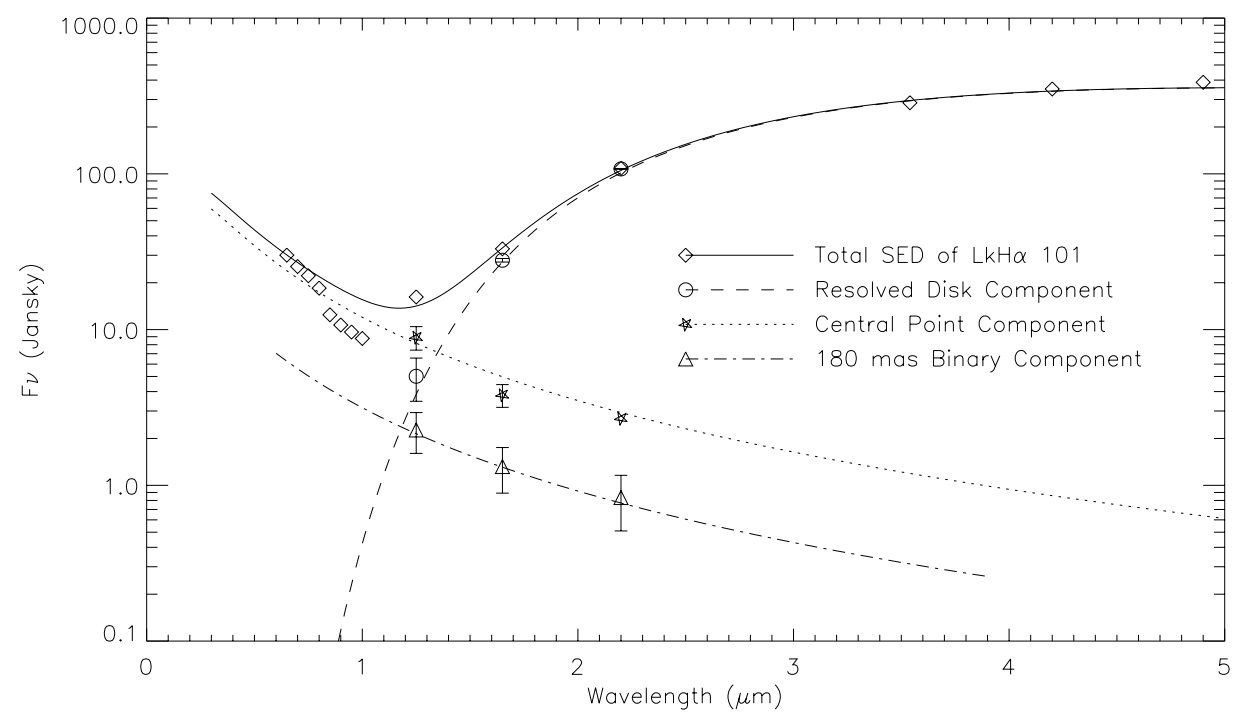

FIG. 7.- SED of $\mathrm{LkH} \alpha 101$ corrected for wavelength-dependent extinction, with $A_{v}=11.2$ (Kelly et al. 1994). Using information from the imaging, the total SED (diamonds) has been divided among the components of the $\mathrm{LkH} \alpha 101$ system: the binary companion (triangles), disk (circles), and central point source (stars). These separate components have also each been modeled by blackbody spectra, as overplotted in the figure (see text for details). 
of circle of brightest emission 21 mas) from the near-IR imaging, and, to within a greater factor $(\sim 4)$, with the midIR size. These discrepancies could probably be taken into account by more complex models in which variation in disk temperature and opacity with radius is allowed.

The central star and binary companion have apparent diameters of 56 and $29 \mu$ as (assuming the 25,000 K temperature). Within the context of this model, we are able to analyze the energetics of the system, finding that $\sim 20 \%$ of the bolometric flux is reprocessed radiation from the dusty disk, while the binary companion contributes $\sim 15 \%$. It is important to emphasize that these conclusions are dependent upon a number of prior assumptions, for example, the visible extinction and the temperatures of the stars (in particular, the temperature of the companion is all but unknown). Although we have taken reasonable estimates for these quantities, the high degree of uncertainty means that the resulting model should be regarded as a plausible exploration rather than an optimized global fit.

It is now possible to use the prior estimates of stellar distance to translate from apparent to absolute quantities. This has been done in Table 5 for the three different distance scales discussed in $\S 4$ 4.2. The absolute luminosities and radii of the central source, the binary companion, and the circumstellar disk are given in consecutive pairs of rows. It is very interesting to note that our 340 pc distance scale results in a stellar luminosity and radius appropriate for a B1 ZAMS star (Panagia 1973), in accord with our expectations from radio photometry and quite close to (although slightly smaller than) our initial model assumption (B0.5; $\left.L=11,000 L_{\odot} ; R=5.1 R_{\odot}\right)$. Note that neither the shorter nor the longer distance scales give this reassuring internal consistency for the model; however, as noted above, different choices of $A_{v}$ or stellar temperature might plausibly result in models that work at these distances.

The value of $R_{\text {dust }}$ given is the diameter of a $1000 \mathrm{~K}$ circular disk that would generate the observed blackbody flux (note this is a simple circular area on the sky). $R_{\text {cav }}$ gives the expected linear size of the observed 21 mas radius circular ridge of brightest emission from the images of Figure 4 . The close agreement between the observed and expected sizes (regardless of distance) gives confidence in the modeling of the SED and our choice of $A_{v}$. It should be emphasized, however, that this is a very simple blackbody model, and a more detailed treatment should try to find a self-consistent thermal profile of the disk.

TABLE 5

Derived Physical Parameters For the LKH $\alpha$ 101 System, Computed For Three Different Possible Stellar Distances

\begin{tabular}{cccc}
\hline \hline & \multicolumn{3}{c}{ DistANCE } \\
\cline { 2 - 4 } PARAMETER & $160 \mathrm{pc}$ & $340 \mathrm{pc}$ & $800 \mathrm{pc}$ \\
\hline$L_{\text {star }}\left(L_{\odot}\right) \ldots \ldots \ldots$. & 1300 & 5900 & 30,000 \\
$R_{\text {star }}\left(R_{\odot}\right) \ldots \ldots \ldots$. & 1.9 & 4.1 & 9.7 \\
$L_{\text {companion }}\left(L_{\odot}\right) \ldots$ & 340 & 1500 & 8500 \\
$R_{\text {companion }}\left(R_{\odot}\right) \ldots$ & 1.0 & 2.1 & 5.0 \\
$L_{\text {dust }}\left(L_{\odot}\right) \ldots \ldots \ldots$. & 270 & 1200 & 6700 \\
$R_{\text {dust }}(\mathrm{AU}) \ldots \ldots \ldots$. & 2.6 & 5.4 & 12.7 \\
$R_{\text {cav }}(\mathrm{AU}) \ldots \ldots \ldots .$. & 3.4 & 7.1 & 16.8 \\
$R_{\text {subl }}(\mathrm{AU}) \ldots \ldots \ldots$. & 2.5 & 5.3 & 12.5 \\
\hline
\end{tabular}

We identify the circular structure as the hot inner walls of the cavity, whose size is likely set primarily by direct radiative dust sublimation from the central star (see, e.g., Tuthill et al. 2001), rather than by the disk-reprocessing or viscousheating processes usually assumed (Lynden-Bell \& Pringle 1974; Hillenbrand et al. 1992). Finally, Table 5 gives $R_{\text {subl }}$, the $1500 \mathrm{~K}$ sublimation radius of isolated dust grains for the system under the assumptions implicit for the three distance scales. Grains surviving at the inner edge would be those best able to reradiate the stellar field, so the assumption of blackbody grains has been made.

It can be seen from the last two lines in the table that the sublimation radius is well matched to the size of the inner cavity, irrespective of the assumed distance. This agreement is encouraging for a simple, radiatively set inner cavity model, although clearly a more detailed treatment could also incorporate effects such as disk self-radiation (Bell 1999). It is interesting to note that material must be continually evaporated at the inner edge in order to maintain this radius; this gas is presumably either ultimately accreted by the central star or swept out into the surrounding $\mathrm{H}$ II region.

\subsection{Parametric Imaging}

As we touched upon in earlier sections, the images produced by the MEM are not unique, nor do the underlying algorithms always represent the best possible approach. In particular, it may be possible to bring extra information to the mapping process, rather than making the assumption that the region of sky to be imaged is most likely flat and featureless.

To take a concrete example, we can infer from Figure 7 that the images in the $K$ band should have a point source at the center contributing $\sim 2.5 \%$ of the flux. Even concentrated into a single, diffraction-limited beam, a feature as weak as this could not be seen amid the bright structure within the resolved disk. This is verified upon examination of images such as those in Figure 4, where no trace of the central point source is seen. However, even bright point sources can fail to appear in MEM maps, as the algorithm will penalize fine structure in the image if alternate, smoother maps can be found which also fit the data.

In this case, we are able to bring our knowledge of the likely presence of a point source into the mapping process. This is done by using a prior, or default map (see, e.g., Monnier et al. 2001). The prior is the map the algorithm will default to in the absence of any constraint from data. We have used a prior map that contains our expected $2.5 \%$ point source at the center, thereby permitting MEM to add fine structure without penalty. The resulting image is given in Figure 8.

The appearance of a compact central component, in accord with our physical expectations for this system, teaches a valuable lesson in caution when interpreting maps made from data taken at close to the diffraction limit. For partially resolved targets, images cannot be definitive, as they have some model-dependent structure. However, this is not to say that the images are in any sense arbitrary. A good example is the almost precisely central location of the point source in the Figure 8 image. Although many trials with priors of various sorts were tried, all cases in which there was a strong point source in the output map had it located at the center. This not only agrees with our physical 


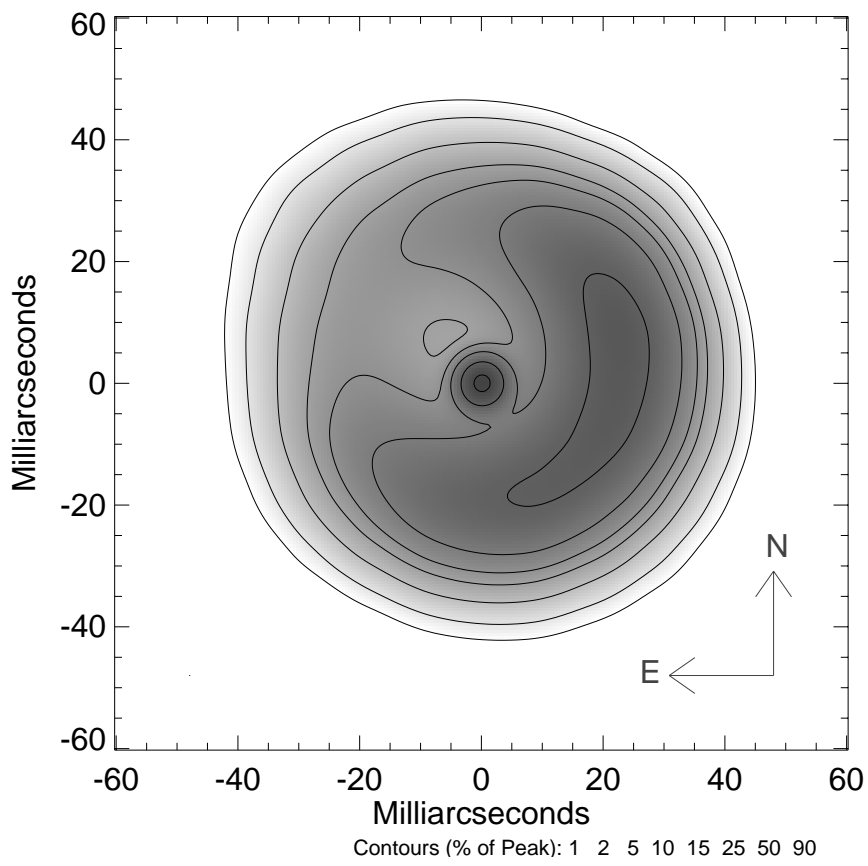

FIG. 8.- Image made with a $2.5 \%$ point-source prior, from data taken in 1998 September through the CH4 filter.

expectations for the system, it is also required by the data. An off-center point source would generate strong telltale visibility and phase signals, as was the case for the 180 mas binary already mapped.

Features for which there is strong evidence in the data will always appear. An example is the limb-brightened ring, which was present, almost unchanged, for all images made. More subtle things, such as the changes in the location of the brightest part of the disk seen in Figure 4, are more open to interpretation. It is apparent that some form of systematic change in the brightness distribution is underway. As the orbital period of material in the disk is about 5 years, assuming the $340 \mathrm{pc}$ distance, it is tempting to interpret the changes as being due to dynamical evolution of the disk. Features such as spiral density perturbations were predicted by Adams, Ruden, \& Shu (1989), and such instabilities may be augmented by the presence of a binary companion or by accounting for self-gravity in a massive disk (see, e.g., Woodward, Tohline, \& Hachisu 1994).

However, the actual depiction in the figure of the bright western crescent splitting to the north and south is modeldependent. For example, the location of the brightest quadrant on the disk was found to be affected by small variations in the location and brightness of the central star. This leads to the possibility that things unrelated to the disk itself, such as varying line-of-sight extinction, may generate changes in appearance of the reconstructed image. We refrain, therefore, from speculation on the detailed cause of these changes.

\section{CONCLUSIONS}

Near-IR images of the Herbig Ae/Be star LkH $\alpha 101$ have been obtained from a multiepoch study utilizing interferometry on the Keck I telescope to obtain information at the diffraction limit (tens of milliarcseconds). The mid-IR UC Berkeley ISI was also able to resolve the system at $11.15 \mu \mathrm{m}$. $\mathrm{LkH} \alpha 101$ presents a resolved circular disk with a central hole or cavity. A relatively blue spectrum binary companion 180 mas to the east-northeast is also reported.

The morphology of the resolved, limb-brightened ring is interpreted as a close-to-face-on viewing angle onto a geometrically thick torus, or circumstellar disk, with a hot inner wall facing the central star. The size of the cavity is consistent with the radiative equilibrium temperature for dust sublimation. A relatively slow increase in apparent diameter of the disk with observing wavelength over a decade from 1.2 to $11.15 \mu \mathrm{m}$ implies circumstellar material with a relatively compact density and/or thermal profile, arguing against classical power-law temperature profiles usually encountered in the literature.

Relative motion of the binary companion, together with simple assumptions on the masses and geometrical projection, favor a likely intermediate distance of around $\sim 340$ pc, although the earlier large (800 pc) and small (160 pc) suggested distance scales (especially the latter) cannot be ruled out.

Combining the interferometry with published photometry enables a decomposition of the SED into three component parts in the infrared. In addition to the 180 mas companion and the resolved disk, we report the probable isolation of a bright, unresolved source at the center of the cavity in the disk, which has been identified as the primary. This is likely an early B star and the source of the photoionizing radiation driving the $\mathrm{H}$ II region. With more detailed radiative transfer modeling and studies at yet higher resolution from the latest generation of optical interferometers, this fascinating system is among the most promising candidates for in-depth study of the workings of a massive young star.

The authors would like to thank Rafael Millan-Gabet, David Hollenbach, Mary Barsony, and Harold Yorke for helpful comments. The image reconstructions presented here were produced by the maximum entropy mapping program VLBMEM, written by Devinder Sivia. This research has made use of the SIMBAD database, operated at CDS, Strasbourg, France, and NASA's Astrophysics Data System Abstract Service. Some of the data presented herein were obtained at the W. M. Keck Observatory, which is operated as a scientific partnership among the California Institute of Technology, the University of California, and the National Aeronautics and Space Administration. The Observatory was made possible by the generous financial support of the W. M. Keck Foundation. The UC Berkeley ISI has been supported in part by grants from the Office of Naval Research (FDN 00014-96-1-0737) and the National Science Foundation (AST 97-31625).
Adams, F. C., Ruden, S. P., \& Shu, F. H. 1989, ApJ, 347, 959

Akeson, R. L., Ciardi, D. R., van Belle, G. T., Creech-Eakman, M. J., \& Lada, E. A. 2000, ApJ, 543, 313

Aspin, C., \& Barsony, M. 1994, A\&A, 288, 849

Barsony, M., Schombert, J. M., \& Kis-Halas, K. 1991, ApJ, 379, 221

\section{REFERENCES}

Barsony, M., Scoville, N. Z., Schombert, J. M., \& Claussen, M. J. 1990, ApJ, 362, 674

Becker, R. H., \& White, R. L. 1988, ApJ, 324, 893

Beckwith, S. V. W., \& Sargent, A. I. 1996, Nature, 383, 139

Bell, K. R. 1999, ApJ, 526, 411 
Bergner, Y. K., Miroshnichenko, A. S., Yudin, R. V., Kuratov, K. S., Mukanov, D. B., \& Shejkina, T. A. 1995, A\&AS, 112, 221

Bittar, J., Tuthill, P., Monnier, J. D., Lopez, B., Danchi, W., \& Stee, P. 2001, A\&A, 368, 197

Böhm, T., \& Catala, C. 1994, A\&A, 290, 167

Chiang, E. I., \& Goldreich, P. 1997, ApJ, 490, 368

Cohen, M. Bieging, J. H. \& Schwartz, P. R. 1982, ApJ, 253, 707

Cohen, M., \& Woolf, N. J. 1971, ApJ, 169, 543

Danchi, W. C., Tuthill, P. G., \& Monnier, J. D. 2001, ApJ, 562, 440

Danen, R. M., Gwinn, C. R., \& Bloemhof, E. E. 1995, ApJ, 447, 391

Dullemond, C. P. 2000, A\&A, 361, L17

Dullemond, C. P., Dominik, C., \& Natta, A. 2001, ApJ, 560, 957

Fuente, A., Neri, R., Martín-Pintado, J., Bachiller, R., Rodríguez-Franco, A. \& Palla, F. 2001, A\&A, 366, 873

Grady, C. A., Woodgate, B., Bruhweiler, F. C., Boggess, A., Plait, P., Lindler, D. J., Clampin, M., \& Kalas, P. 1999, ApJ, 523, L151

Hale, D. D. S., et al. 2000, ApJ, 537, 998

Haniff, C. A., \& Buscher, D. F. 1992, J. Opt. Soc. Am. A, 9, 203

Harris, S. 1976, MNRAS, 174, 601

Hartmann, L., Kenyon, S. J., \& Calvet, N. 1993, ApJ, 407, 219

Herbig, G. H. 1956, PASP, 68, 353

$$
\text { 1971, ApJ, 169, } 537
$$

Hillenbrand, L. A., Strom, S. E., Vrba, F. J., \& Keene, J. 1992, ApJ, 397, 613

Hinz, P. M., Hoffmann, W. F., \& Hora, J. L. 2001, ApJ, 561, L131

Hollenbach, D. Johnstone, D. Lizano, S. \& Shu, F. 1994, ApJ, 428, 654

Hou, J., Jiang, D., \& Fu, C. 1997, A\&A, 327, 725

Kant, I. 1755, Allgemeine Naturgeschichte und Theorie des Himmels (Leipzig: Engelmann)

Kelly, D. M., Rieke, G. H. \& Campbell, B. 1994, ApJ, 425, 231

Kessel, O., Yorke, H. W., \& Richling, S. 1998, A\&A, 337, 832

Koresko, C. D. 1998, ApJ, 507, L145

Leinert, C., Haas, M., Ábráham, P., \& Richichi, A., 2001, A\&A, 375, 927

Lynden-Bell, D., \& Pringle, J. E. 1974, MNRAS, 168, 603

Mannings, V., \& Sargent, A. I. 1997, ApJ, 490, 792
Mannings, V., \& Sargent, A. I. 2000, ApJ, 529, 391

Mathis, J. S. 1990, ARA\&A, 28, 37

Matthews, K., Ghez, A. M., Weinberger, A. J., \& Neugebauer, G. 1996, PASP, 108,615

McCaughrean, M. J., \& O’Dell, C. R. 1996, AJ, 111, 1977

McGregor, P. J., Persson, S. E., \& Cohen, J. G. 1984, ApJ, 286, 609

Millan-Gabet, R., Schloerb, F. P., \& Traub, W. A. 2001, ApJ, 546, 358

Millan-Gabet, R., Schloerb, F. P., Traub, W. A., Malbet, F., Berger, J. P., \& Bregman, J. D. 1999, ApJ, 513, L131

Miroshnichenko, A., Ivezić, Z., \& Elitzur, M. 1997, ApJ, 475, L41

Miroshnichenko, A., Ivezić, Z., Vinković, D., \& Elitzur, M. 1999, ApJ, 520, L115

Monnier, J. D., et al. 2001, BAAS, 198, 63.02

Narayan, R., \& Nityananda, R. 1986, ARA\&A, 24, 127

Natta, A., Prusti, T., Neri, R., Wooden, D., Grinin, V. P., \& Mannings, V. 2001, A\&A, 371, 186

O’Dell, C. R., Wen, Z., \& Hu, X. 1993, ApJ, 410, 696

Panagia, N. 1973, AJ, 78, 929

Pezzuto, S., Strafella, F., \& Lorenzetti, D. 1997, ApJ, 485, 290

Redman, R. O., Kuiper, T. B. H., Lorre, J. J., \& Gunn, J. E. 1986, ApJ, 303,300

Rudy, R. J., Erwin, P., Rossano, G. S., \& Puetter, R. C. 1991, ApJ, 383, 344

Shu, F. H., Tremaine, S., Adams, F. C., \& Ruden, S. P. 1990, ApJ, 358, 495

Simon, M., \& Cassar, L. 1984, ApJ, 283, 179

Sivia, D. S. 1987, Ph.D. thesis, Cambridge Univ.

Skilling, J., \& Bryan, R. K. 1984, MNRAS, 211, 111

Smith, B. A. \& Terrile, R. J. 1984, Science, 226, 1421

Stine, P. C., \& O’Neal, D. 1998, AJ, 116, 890

Thompson, R. I., Strittmatter, P. A., Erickson, E. F., Witteborn, F. C., \& Strecker, D. W. 1977, ApJ, 218, 170

Tuthill, P. G., Monnier, J. D., \& Danchi, W. C. 2001, Nature, 409, 1012

Tuthill, P. G., Monnier, J. D., Danchi, W. C., Wishnow, E. H., \& Haniff, C. A. 2000, PASP, 112, 555

Woodward, J. W., Tohline, J. E., \& Hachisu, I. 1994, ApJ, 420, 247

Yorke, H. W., \& Welz, A. 1996, A\&A, 315, 555 